




\title{
Secant spaces and syzygies of special line bundles on curves
}

\author{
Marian Aprodu and Edoardo Sernesi
}

On a special line bundle $L$ on a projective curve $C$ we introduce a geometric condition called $\left(\Delta_{q}\right)$. When $L=K_{C}$, this condition implies $\operatorname{gon}(C) \geq q+2$. For an arbitrary special $L$, we show that $\left(\Delta_{3}\right)$ implies that $L$ has the well-known property $\left(M_{3}\right)$, generalising a similar result proved by Voisin in the case $L=K_{C}$.

\section{Introduction}

In this paper we introduce some new geometric methods in the study of the Koszul cohomology groups of a projective curve with coefficients in an invertible sheaf. The basic set-up is as follows.

Let $C$ be a smooth complex projective curve of genus $g$, and $L$ a very ample line bundle of degree $d$ on $C$ with $h^{0}(C, L)=r+1$. Consider a coherent sheaf $\mathscr{F}$ on $C$ and let $V=H^{0}(C, L)$; one has natural complexes of vector spaces

$\bigwedge^{p+1} V \otimes H^{0}\left(\mathscr{F} \otimes L^{q-1}\right) \longrightarrow \bigwedge^{p} V \otimes H^{0}\left(\mathscr{F} \otimes L^{q}\right) \longrightarrow \bigwedge^{p-1} V \otimes H^{0}\left(\mathscr{F} \otimes L^{q+1}\right)$,

whose cohomology $K_{p, q}(C, \mathscr{F} ; L)$ is called the $(p, q)$ (mixed) Koszul cohomology group of $C$ with respect to $\mathscr{F}$ and $L$. These vector spaces give information about the minimal resolution of the graded module

$$
\gamma(C, \mathscr{F} ; L)=\bigoplus_{k} H^{0}\left(\mathscr{F} \otimes L^{k}\right)
$$

over the symmetric (polynomial) algebra $R=S^{*} V$ in a well-known way (see [Aprodu and Nagel 2010]). The most important cases are obtained for $\mathscr{F}_{F}=O_{C}$; the corresponding graded $R$-module $\bigoplus_{k} H^{0}\left(L^{k}\right)$ is denoted by $\gamma(C ; L)$ and its Koszul

\footnotetext{
Aprodu thanks the University of Trento and the Dipartimento di Matematica e Fisica, Università degli Studi Roma Tre for hospitality during the preparation of this work. Sernesi is grateful to the IMAR and to the University of Trento for hospitality during the preparation of this work. The authors have been partly supported by a RIP of CIRM-Trento. Aprodu was partly supported by the grant PN-II-ID-PCE-2012-4-0156. Sernesi was partly supported by the project MIUR-PRIN 2010/11 Geometria delle varietà algebriche.
}

MSC2010: primary 14N05; secondary 14N25, 14M12.

Keywords: projective curves, Brill-Noether theory, syzygies, secant loci. 
cohomology groups by $K_{p, q}(C ; L)$. The choice $L=K_{C}$ is of central importance, and its study is at the origin of several results and conjectures on this subject. The guiding notions are the so-called properties $\left(N_{p}\right)$.

Definition 1.1. The line bundle $L$ has property $\left(N_{0}\right)$ if and only if the natural restriction map $\rho: R \longrightarrow \gamma(C ; L)$ is surjective, i.e., $L$ is normally generated. For $p \geq 1$, we say that the bundle $L$ satisfies the property $\left(N_{p}\right)$ if and only if it is normally generated and $K_{i, j}(C ; L)=0$ for all $j \neq 1$ and all $1 \leq i \leq p$.

Roughly speaking, $\left(N_{p}\right)$ holds if and only if the minimal resolution of $\gamma(C ; L)$ behaves nicely up to the $p$-th step. These notions have provided an excellent motivation on these problems in two important cases, namely in the case $L=K_{C}$ and in the case $\operatorname{deg}(L) \gg 0$. As an example, we recall the following:

Theorem 1.2 [Green and Lazarsfeld 1985]. If $\operatorname{deg}(L) \geq 2 g+1+p$, then L has property $\left(N_{p}\right)$. If $\operatorname{deg}(L) \geq 2 g+p$, then $L$ has property $\left(N_{p}\right)$ unless $C$ is hyperelliptic or L embeds $C$ in $\mathbb{P}^{g+p}$ with $a(p+2)$-secant p-plane.

Property $\left(N_{p}\right)$ for special line bundles is also highly interesting; the study of possible divisorial cases in the moduli space of pairs $(C, L)$, for special line bundles $L$ with $h^{1}(C, L)>1$ and which fail property $\left(N_{p}\right)$, has revealed a whole class of counterexamples for the slope conjecture [Farkas 2009]. However, the relations between the properties $\left(N_{p}\right)$ and the geometry of the projective model $\varphi_{L}(C)$ when $L$ is a special line bundle different from $K_{C}$, especially if $h^{1}(C, L)>1$, remain somewhat mysterious. Already $\left(N_{0}\right)$ and $\left(N_{1}\right)$ have escaped a systematic classification for obvious reasons: normal generation and ideal generation of special projective curves behave essentially wildly and it is therefore very difficult to get even a conjectural picture of how the resolution of $\gamma(C ; L)$ might look like (see [Aprodu and Nagel 2010, Section 4.4] for a short discussion).

A possible solution comes from the study of other properties of $\gamma(C ; L)$, called $\left(M_{q}\right)$, which were introduced in [Green and Lazarsfeld 1986] for $q \geq 1$. We shall work with a slightly weaker condition than there, in the spirit of [Ehbauer 1994].

Definition 5.3. The line bundle $L$ has property $\left(M_{q}\right)$ if $K_{n, 1}(C ; L)=0$ for all $n \geq r-q$.

These are properties enjoyed by the tail of the resolution of $\gamma(C ; L)$; i.e., property $\left(M_{q}\right)$ holds for $L$ if the resolution of $\gamma(C ; L)$ has a nice behaviour at the last $q$ steps. Another, perhaps more suggestive, point of view consists of considering the resolution of the module $\gamma\left(C, K_{C} ; L\right)$. Since it is dual to $\gamma(C ; L)$, properties $\left(M_{q}\right)$ for $L$ correspond to nice behaviour of the head of the resolution of $\gamma\left(C, K_{C} ; L\right)$. In a landmark paper, Petri [1925] had already focused his attention on the module 
$\gamma\left(C, K_{C} ; L\right)$ when $L$ is special. Arbarello and Sernesi [1978] showed that Petri's analysis contains a proof of $\left(M_{1}\right)$ for all $L$ on a nonrational curve $C$ and a characterisation of the validity of $\left(M_{2}\right)$ when $L$ is special. Note that when $L=K_{C}$ the self-duality of the resolution of $\gamma\left(C ; K_{C}\right)$ implies that property $\left(M_{q}\right)$ is equivalent to property $\left(N_{q-1}\right)$, so the result discussed in [Arbarello and Sernesi 1978] generalises Petri's celebrated analysis of the ideal of the canonical model of a nonhyperelliptic curve (see [Saint-Donat 1973]).

The present paper is devoted to the study of $\left(M_{3}\right)$ for a special $L$. This property has been already studied and characterised for $L=K_{C}$ by Schreyer [1991], by Voisin [1988] and when $\operatorname{deg}(L) \gg 0$ by Ehbauer [1994]. The main issue in considering the case of any special line bundle, not considered by them, is to find natural geometric conditions on $C$ and $L$. We introduce the following definition:

Definition 2.3. Assume that $r \geq 4$, and let $2 \leq q \leq 1+r / 2$. We say that a reduced effective divisor $D=x_{1}+\cdots+x_{r-q+2}$ on $C$ satisfies condition $\left(\Delta_{q}\right)$ with respect to $L$ if the following conditions are satisfied:

(a) $h^{0}(L(-D))=q$.

(b) $L(-D)$ is basepoint-free.

(c) $h^{0}\left(L\left(-D+x_{i}\right)\right)=h^{0}(L(-D))$ for all $i=1, \ldots, r-q+2$.

In the case $L=K_{C}$, a divisor $D$ satisfies condition $\left(\Delta_{q}\right)$ if it defines a primitive $g_{g-q+1}^{1}$. In general, $D$ defines an $(r-q)$-plane in $\mathbb{P}^{r}$ which is precisely $(r-q+2)$ secant to $\varphi_{L}(C) \subset \mathbb{P}^{r}$. This condition has appeared in [Green and Lazarsfeld 1985] in the case $q=2$ and in [Voisin 1988], where it is called $\left(H_{1}\right)$, in the case $q=3$. In both cases they have proved to be the key for $\left(M_{2}\right)$ to hold for $K_{C}$ (equivalent to Petri's theorem) and for $\left(M_{3}\right)$ to hold for $K_{C}$, respectively. More precisely, a divisor $D=x_{1}+\cdots+x_{g-1}$ satisfying condition $\left(\Delta_{2}\right)$ for $K_{C}$ defines a primitive $g_{g-1}^{1}$, and the existence of such a $D$ can be seen to be equivalent to $C$ being not exceptional, i.e., to $\operatorname{Cliff}(C) \geq 2$ : this is how Green and Lazarsfeld arrive at Petri's theorem involving $\left(\Delta_{2}\right)$ and using the Mumford-Martens theorem. On the other hand, via an elaborate analysis, Voisin showed for $g \geq 11$ that $\left(\Delta_{3}\right)$ plus $\operatorname{Cliff}(C) \geq 3$ imply that a general projection in $\mathbb{P}^{5}$ of the canonical model of $C$ satisfies $\left(M_{3}\right)$. It is interesting to note that this is achieved by excluding in particular that the projected curve lies in certain surfaces that are intersection of quadrics in $\mathbb{P}^{5}$. Here one cannot but observe the analogy with the way Ehbauer [1994] proved $\left(M_{3}\right)$ for $L$ such $\operatorname{deg}(L) \gg 0$ : while his method is different from Voisin's, he is led to consider the same list of surfaces.

Our main result involves condition $\left(\Delta_{3}\right)$ plus a transversality condition as the key hypothesis. Specifically, we prove the following: 
Theorem 5.4. Assume $g \geq 14, r \geq 5$, that $L$ is very ample and special of degree $\geq r+13$, that each component of the locus of $(r-1)$-secant $(r-3)$-planes has the expected dimension $r-4$, and that the general such $(r-3)$-plane in each component satisfies $\left(\Delta_{3}\right)$ with respect to $L$. Then $L$ satisfies $\left(M_{3}\right)$ unless $\operatorname{Cliff}(C) \leq 2$.

The relation between condition $\left(\Delta_{3}\right)$ and the vanishing of the $K_{i, 1}(C ; L)$ for all $i \geq r-3$ is roughly the following: Nonzero elements of the $K_{i, 1}(C ; L)$ can be seen to correspond to certain subvarieties containing the curve $\varphi_{L}(C) \subset \mathbb{P}^{r}$ and defined by quadrics. On the other hand, the existence of divisors satisfying $\left(\Delta_{3}\right)$ plays the role of a generality condition which prevents the curve from being contained in such a variety. This simple contradiction works quite efficiently once the curve is projected in $\mathbb{P}^{5}$, and that's how we prove the theorem. Note that the condition $\operatorname{Cliff}(C) \geq 3$ cannot be removed, as easy examples show.

For higher $q$ we have a similar contradiction. But the verification that $\left(M_{q}\right)$ holds once hypotheses similar to those of the theorem are satisfied becomes much more involved as $q \geq 4$, and would require a classification of certain classes of varieties that is not yet available.

It is interesting to note that in Theorem 1.2 the existence of secant spaces is related to the exceptions to the validity of $\left(N_{p}\right)$; hence, it is not satisfied in general. On the other hand, in Theorem 5.4 the existence of secant spaces, implied by condition $\left(\Delta_{3}\right)$, is satisfied in general.

A final note in the case $L=K_{C}$. The condition $\operatorname{Cliff}(C) \geq 2$ already implies the existence of divisors satisfying $\left(\Delta_{2}\right)$. Similarly, the use of condition $\left(\Delta_{3}\right)$ made by Voisin [1988] plays a role in the proof, but is not required for the validity of $\left(M_{3}\right)$ : all that is required is that $\operatorname{Cliff}(C) \geq 3$; in fact the main difficulty in that work consists of proving that $\operatorname{Cliff}(C) \geq 3$ implies the existence of $D$ satisfying $\left(\Delta_{3}\right)$. This suggests, more generally, that $\operatorname{Cliff}(C) \geq q$ might imply the existence of divisors $D$ satisfying $\left(\Delta_{q}\right)$ with respect to $K_{C}$.

The paper is organised as follows. In Section 2 we introduce the main condition $\left(\Delta_{q}\right)$ and study its general properties. In Section 3 we specialise to the case of canonical curves. In Section 4 we relate condition $\left(\Delta_{q}\right)$ to the geometry of the curve in $\mathbb{P}^{r}$, and in Section 5 we recall the definition of syzygy schemes and prove Theorem 5.4.

\section{The condition $\left(\Delta_{q}\right)$}

2A. Secant loci. For any $n \geq 1$, we denote by $C_{n}$ the $n$-th symmetric product of $C$ and by $\Xi_{n} \subset C \times C_{n}$ the universal divisor. Let

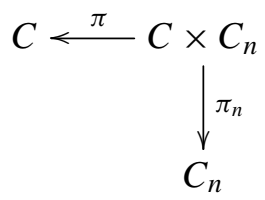


be the projections. For any globally generated line bundle $L$ on $C$, the sheaf on $C_{n}$

$$
E_{L}:=\pi_{n *}\left(\pi^{*} L \otimes \mathcal{O}_{\Xi_{n}}\right)
$$

is locally free of rank $n$ and is called the secant bundle of $L$. We have a homomorphism of locally free sheaves on $C_{n}$

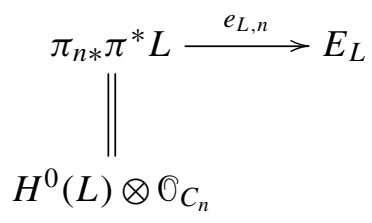

Note that $e_{L, n}$ is generically surjective if $n \leq r$.

We will denote by $V_{n}^{k}(L) \subset C_{n}$ the closed subscheme defined by the condition

$$
\operatorname{rank}\left(e_{L, n}\right) \leq k .
$$

Standard facts about determinantal subschemes (see, for example, [Arbarello et al. 1985]) imply that if nonempty, then $V_{n}^{k}(L)$ has dimension $\geq n-(r+1-k)(n-k)$, which is the expected dimension.

Of special interest are the cases $k=n-1$. The scheme $V_{n}^{n-1}(L)$ is supported on the set of $D \in C_{n}$ which do not impose independent conditions on $L$, and its expected dimension is $2 n-r-2$. If $n=r$, we can prove the following:

Lemma 2.1. If $r \geq 4$ then $V_{r}^{r-1}(L)$ is nonempty and of pure dimension $r-2$.

Proof. Let $\Sigma$ be a nonempty component of $V_{r}^{r-1}(L)$ with $\operatorname{codim}(\Sigma) \leq 1$, i.e., with $\operatorname{dim}(\Sigma) \geq r-1$. Consider the morphisms

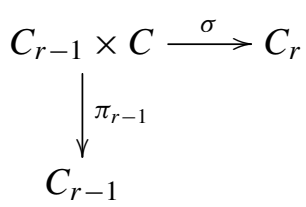

Then $\pi_{r-1}\left(\sigma^{-1}(\Sigma)\right)=C_{r-1}$. This implies that if $x_{1}, \ldots, x_{r-1} \in C$ are general points then the pencil $\left|L\left(-x_{1}-\cdots-x_{r-1}\right)\right|$ has basepoints, which is impossible. Therefore $V_{r}^{r-1}(L)$ has pure dimension $r-2$.

For the same reason, if $A=x_{1}+\cdots+x_{r-2}$ is a general effective divisor of degree $r-2$, then $L(-A)$ is basepoint-free and not composed with an involution. The plane curve $\Gamma:=\varphi_{L(-A)}(C) \subset \mathbb{P}^{2}$ is singular and birational to $C$. Letting $x_{r-1}, x_{r} \in$ $C$ be such that $\varphi_{L(-A)}\left(x_{r-1}\right)=\varphi_{L(-A)}\left(x_{r}\right)$ is a singular point of $\Gamma$, the divisor $x_{1}+\cdots+x_{r-2}+x_{r-1}+x_{r}$ belongs to $V_{r}^{r-1}(L)$, which shows nonemptiness.

Let us record the following useful fact, which is a direct generalisation of [Arbarello et al. 1985, Lemma 1.7, p. 163]: 
Lemma 2.2. Assume that $q \geq 2, r-q+2 \geq 4$ and $V_{r-q+2}^{r-q+1}(L) \neq \varnothing$. Then no irreducible component of $V_{r-q+2}^{r-q+1}(L)$ is contained in $V_{r-q+2}^{r-q}(L)$.

Proof. Let $D=x_{1}+\cdots+x_{r-q+2}$ be a general element in a component of $V_{r-q+2}^{r-q+1}(L)$. Assume by contradiction that $D \in V_{r-q+2}^{r-q}(L)$. Then $\operatorname{dim}\langle D\rangle \leq r-q-1$. We may assume that $\langle D\rangle=\left\langle x_{1}+\cdots+x_{r-q+1}\right\rangle$. Then for a general $x \in C$ we have $\operatorname{dim}\left\langle x_{1}+\cdots+x_{r-q+1}+x\right\rangle \leq r-q$ and therefore $x_{1}+\cdots+x_{r-q+1}+x \in V_{r-q+2}^{r-q+1}(L)$. To conclude, note that $x_{1}+\cdots+x_{r-q+1}+x, D$ belong to the same component of $V_{r-q+2}^{r-q+1}(L)$ and $\operatorname{dim}\langle D\rangle<\operatorname{dim}\left\langle x_{1}+\cdots+x_{r-q+1}+x\right\rangle$, contradicting the generality of $D$.

A consequence of Lemma 2.2 is that the locally closed subscheme $S_{r-q+2}(L) \subset$ $C_{r-q+2}$ defined as

$$
S_{r-q+2}(L):=V_{r-q+2}^{r-q+1}(L) \backslash V_{r-q+2}^{r-q}(L)
$$

is dense in any irreducible component of $V_{r-q+2}^{r-q+1}(L)$. In particular, any property which is satisfied by general divisors in any irreducible component of $V_{r-q+2}^{r-q+1}(L)$ is also valid for $S_{r-q+2}(L)$. Note that the expected dimension is $r-2 q+2$ in this case. For the particular case $q=2$, Lemma 2.1 shows that the dimension of $S_{r}(L)$ coincides with the expected dimension $r-2$.

2B. Condition $\left(\Delta_{q}\right)$. We introduce our basic condition:

Definition 2.3. Assume that $r \geq 4$, and let $2 \leq q \leq 1+r / 2$. We say that a reduced effective divisor $D=x_{1}+\cdots+x_{r-q+2}$ on $C$ satisfies condition $\left(\Delta_{q}\right)$ with respect to $L$ if the following conditions are satisfied:

(a) $h^{0}(L(-D))=q$.

(b) $L(-D)$ is basepoint-free.

(c) $h^{0}\left(L\left(-D+x_{i}\right)\right)=h^{0}(L(-D))$ for all $i=1, \ldots, r-q+2$.

In terms of projective geometry, the conditions defining $\left(\Delta_{q}\right)$ can be rephrased as follows:

(a) The linear span $\langle D\rangle \subset \mathbb{P}^{r}$ is an $(r-q)$-plane.

(b) $\langle D\rangle \cap C=\operatorname{Supp}(D)$.

(c) $x_{1}, \ldots, x_{r-q+2}$ are in linearly general position in $\langle D\rangle$ (but not in $\mathbb{P}^{r}$ of course); i.e., $\left\langle D-x_{i}\right\rangle=\langle D\rangle$ for all $i$.

In terms of symmetric products, the conditions defining $\left(\Delta_{q}\right)$ correspond to the following:

(a) $D \in S_{r-q+2}(L)$.

(b) $\{D\}+C \subset S_{r-q+3}(L)$. 
(c) $D \notin \operatorname{Im}\left\{V_{r-q+1}^{r-q}(L) \times C \rightarrow C_{r-q+2}\right\}$.

Note that, from Lemma 2.2, a general point in any irreducible component of $V_{r-q+2}^{r-q+1}(L)$ satisfies condition (a). Clearly, divisors $D=x_{1}+\cdots+x_{r-q+2}$ as in Definition 2.3 fill an open subset of $S_{r-q+2}(L)$.

Proposition 2.4. Assume that $L$ is special and embeds $C$ with $a(r-q+2)$-secant $(r-q)$-plane $\langle D\rangle \subset \mathbb{P}^{r}$. Then $h^{0}\left(\mathcal{O}_{C}(D)\right) \leq 2$.

Proof. Assume that $L=K_{C}(-B)$, and set $r_{B}:=h^{0}\left(\mathscr{O}_{C}(B)\right)-1=h^{1}(L)-1$. From the Riemann-Roch theorem applied to $L$, we obtain $\operatorname{deg}(B)=r_{B}-r+g-1$, and hence $\operatorname{deg}(B+D)=g-q+r_{B}+1$. From Riemann-Roch applied to $L(-D)$, we obtain $h^{0}\left(\mathscr{O}_{C}(B+D)\right)=r_{B}+2$. Since the addition map of divisors $|B| \times|D| \rightarrow$ $|B+D|$ is finite on its image, it follows that $\operatorname{dim}|D| \leq 1$.

Remark 2.5. (i) A divisor $D$ satisfies $\left(\Delta_{q}\right)$ with respect to $K_{C}$ if and only if $|D|$ is a primitive $g_{g-q+1}^{1}$. In particular, $\left(\Delta_{2}\right)$ is equivalent to $|D|$ being a primitive $g_{g-1}^{1}$ on $C$, and such a $D$ does not exist if and only if $C$ is trigonal or a nonsingular plane quintic (see [Green and Lazarsfeld 1985]). Note that hyperelliptic curves are excluded automatically by our assumptions if $L=K_{C}$. We shall treat the canonical case in a separate section.

(ii) If $L$ is nonspecial of degree $d=g+r \geq 2 g$, then there is no divisor $D \in C_{r-g+1}$ satisfying condition $\left(\Delta_{g+1}\right)$ with respect to $L$. In fact this would imply that $L(-D)$ is basepoint-free of degree $(g+r)-(r-g+1)=2 g-1$ and dimension $r-(r-g)=g$, and this is impossible. If $g=1$, this means that no $D \in C_{r}$ satisfies $\left(\Delta_{2}\right)$ with respect to $L$ : in fact, $C \subset \mathbb{P}^{r}$ has degree $r+1$ and any $r$ distinct points of $C$ are independent.

Terminology. Assume $L$ to be special and very ample, $h^{0}(L)=r+1$, and let $2 \leq q \leq r-1$. It is convenient to introduce the following:

- We say that condition $\left(\Delta_{q}\right)$ holds on a component $V$ of $V_{r-q+2}^{r-q+1}(L)$ if the general element $D \in V$ satisfies $\left(\Delta_{q}\right)$ with respect to $L$. We say that $\left(\Delta_{q}\right)$ holds on $C$ with respect to $L$ if it holds on every component of $V_{r-q+2}^{r-q+1}(L)$.

- We say that $\left(\Delta_{q}\right)$ holds on $C$ with respect to $L$ in the strong sense if it holds, and moreover all components of $V_{r-q+2}^{r-q+1}(L)$ have dimension equal to the expected dimension $r-2 q+2$. A necessary condition for this to happen is that $r \geq 2 q-2$.

- When we say " $\operatorname{dim}(Z)=d$ ", we mean that each irreducible component of $Z$ has dimension $d$.

Most of our results are proved only under the assumption that $\left(\Delta_{q}\right)$ holds in the strong sense. 
Proposition 2.6. Assume that $\operatorname{dim}\left(V_{r-q+2}^{r-q+1}(L)\right)=r-2 q+2$. Then $\left(\Delta_{q}\right)$ holds on $C$ with respect to $L$ in the strong sense if and only if the following conditions are satisfied:

(1) $\operatorname{dim}\left(V_{r-q+3}^{r-q+1}(L)\right) \leq r-2 q+1$.

(2) $\operatorname{dim}\left(V_{r-q+1}^{r-q}(L)\right)=r-2 q$.

Proof. Note that the expected dimension of the locus $V_{r-q+3}^{r-q+1}(L)$ is $r-3 q+3 \leq$ $r-2 q+1$.

The proof relies on the observation that any map defined by addition of divisors is finite on its image. Assume $\operatorname{dim}\left(V_{r-q+3}^{r-q+1}(L)\right) \leq r-2 q+1$ and $\operatorname{dim}\left(V_{r-q+1}^{r-q}(L)\right)=$ $r-2 q$. Let $D \in S_{r-q+2}(L)$ be a general element in an irreducible component. Then by definition $h^{0}(L(-D))=q$, hence condition (a) from Definition 2.3 is satisfied. We prove that $L(-D)$ has no basepoints, i.e., condition (b). Suppose that $x$ is a basepoint of $L(-D)$; then $D+x$ is in $V_{r-q+3}^{r-q+1}(L)$ and depends on $r-2 q+2$ parameters, contradicting the assumption on $\operatorname{dim}\left(V_{r-q+3}^{r-q+1}(L)\right)$. We have seen that condition (c) is equivalent to $D \notin \operatorname{Im}\left\{V_{r-q+1}^{r-q}(L) \times C \rightarrow C_{r-q+2}\right\}$. By the dimensionality assumptions, the image of the addition map cannot fill a dense set of a component of $S_{r-q+2}(L)$.

Conversely, assume that $\left(\Delta_{q}\right)$ hold on $C$ with respect to $L$ in the strong sense. Suppose that $V_{r-q+1}^{r-q}(L)$ has a component $Z$ with $\operatorname{dim}(Z) \geq r-2 q+1$. Then by the dimensionality hypothesis, the image of the set $Z+C$ inside $V_{r-q+2}^{r-q+1}(L)$ must fill a component, and all its points violate $\left(\Delta_{q}\right)$. If there is a component $Y$ of $V_{r-q+3}^{r-q+1}(L)$ having dimension $\geq r-2 q+2$, then a general element $D^{\prime} \in Y$ can be written as $D^{\prime}=D+x$, where, again by the dimensionality assumption, $D$ must fill a component of $V_{r-q+2}^{r-q+1}(L)$. From the definition, $D$ fails property (b) of $\left(\Delta_{q}\right)$, a contradiction.

Remark 2.7. Recall that in the case $q=2$ the dimension of the locus $V_{r}^{r-1}(L)$ equals the expected dimension $r-2$ (Lemma 2.1) but it can be reducible: when $L=K_{C}$ and $g \geq 6$ this happens precisely when $C$ is either trigonal or bielliptic (see [Teixidor i Bigas 1984]). In the trigonal case $V_{g-1}^{g-2}\left(K_{C}\right)$ has two components, and in both of them $\left(\Delta_{2}\right)$ does not hold. In the bielliptic case $\left(\Delta_{2}\right)$ holds in one component but not in the other. A characterisation of the pairs $(C, L)$ for which $V_{r}^{r-1}(L)$ is reducible is unknown to us when $L$ is arbitrary.

Lemma 2.8. Assume $r \geq 5$ and $2 \leq q \leq(r+1) / 2$. Assume that $\left(\Delta_{q}\right)$ holds on $C$ with respect to $L$ in the strong sense. Then, for every general $x \in C,\left(\Delta_{q}\right)$ holds on $C$ with respect to $L(-x)$ in the strong sense.

Proof. As noted before, it suffices to prove the same statement for the locally closed subschemes $S_{r-q+1}$. Let $x \in C$ be a point such that, for each irreducible component of $S_{r-q+2}(L)$, it is not in the support of all divisors of that component and it is in 
the support of some divisor in it that satisfies $\left(\Delta_{q}\right)$ with respect to $L$. We have a diagram of spaces and maps

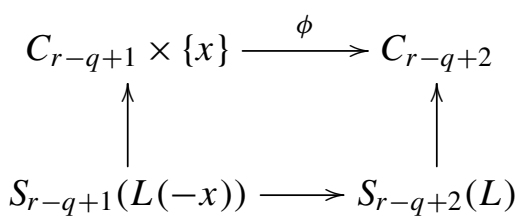

where all the maps are inclusions. Let $\Sigma \subset S_{r-q+1}(L(-x))$ be an irreducible component. Assume that $\operatorname{dim}(\Sigma) \geq r-2 q+2$. Then $\phi(\Sigma)$ is a component of $\overline{S_{r-q+2}(L)}$ and all divisors in $\phi(\Sigma)$ contain $x$ in their support. This contradicts our assumptions. The second possibility is that $\operatorname{dim}(\Sigma)=r-2 q+1$ and that all divisors $D \in \Sigma$ do not satisfy $\left(\Delta_{q}\right)$ with respect to $L(-x)$. Then $\phi(\Sigma) \subset \overline{S_{r-q+2}(L)}$ and all $D+x \in \phi(\Sigma)$ do not satisfy $\left(\Delta_{q}\right)$ with respect to $L$. Since this condition is satisfied for a general choice of $x \in C$, we deduce that there is a component of $\overline{S_{r-q+2}(L)}$ with no elements satisfying $\left(\Delta_{q}\right)$ with respect to $L$, a contradiction.

\section{The case $L=K_{C}$}

In this case the notation specialises as follows:

- $V_{g-q+1}^{g-q}\left(K_{C}\right)=C_{g-q+1}^{1}$.

- $S_{g-q+1}\left(K_{C}\right)=C_{g-q+1}^{1} \backslash C_{g-q+1}^{2}$.

- The expected dimension of $V_{g-q+1}^{g-q}\left(K_{C}\right)$ is $g-2 q+1$.

- A divisor $D \in C_{g-q+1}^{1}$ satisfies $\left(\Delta_{q}\right)$ with respect to $K_{C}$ for some $q \geq 2$ if and only if it defines a primitive $g_{g-q+1}^{1}$, i.e., it is complete, basepoint-free and the residual is also basepoint-free.

For brevity, when in this section we say that a condition $\left(\Delta_{q}\right)$ is satisfied, we assume implicitly "with respect to $K_{C}$ ".

The condition $\left(\Delta_{q}\right)$ is well defined in the range $2 \leq q \leq g-1$. When $[(g-1) / 2]<$ $q \leq g-1$, the existence of a $D \in C_{g-q+1}$ satisfying $\left(\Delta_{q}\right)$ is equivalent to the existence of a primitive $g_{g-q+1}^{1}$ with $g-q+1<(g+3) / 2$, and therefore $C$ becomes more and more special as $q$ grows, because its gonality decreases. On the other hand, when $2 \leq q \leq[(g-1) / 2]$, the condition that there exists $D$ satisfying $\left(\Delta_{q}\right)$ should imply that $\operatorname{Cliff}(C) \geq q$ (this is true for $q=2,3$, see the Remark 3.4 below). In this range, if this implication is true then the existence of a $D \in C_{g-q+1}$ satisfying $\left(\Delta_{q}\right)$ implies that $C$ is more and more general as $q$ grows. We are able to clarify this, assuming only that $C$ has Clifford dimension 1. 
Proposition 3.1. Assume $g \geq 2 q+1$ and $q \geq 2$. Consider the following conditions:

(i) The condition $\left(\Delta_{q}\right)$ holds on $C$ in the strong sense.

(ii) $C \subset \mathbb{P}^{g-1}$ is not contained in a $q$-dimensional variety of minimal degree $g-q$.

(iii) For all $1 \leq e \leq q$, there does not exist a $\bar{D} \in C_{e+1}$ satisfying $\left(\Delta_{g-e}\right)$.

(iv) $\operatorname{gon}(C) \geq q+2$.

We have (i) $\Longrightarrow$ (ii) $\Longleftrightarrow$ (iii) $\Longleftrightarrow$ (iv).

Proof. (iv) $\Longleftrightarrow$ (iii). gon $(C)<q+2$ if and only if there exists a primitive $g_{e+1}^{1}$ for some $1 \leq e \leq q$, and this is equivalent to the existence of $\bar{D} \in C_{e+1}$ satisfying $\left(\Delta_{g-e}\right)$.

(ii) $\Longleftrightarrow$ (iii). The existence of a primitive $g_{e+1}^{1}$ for some $1 \leq e \leq q$ is equivalent to the existence of $A \in W_{q+1}^{1} \backslash W_{q+1}^{2}$, possibly with basepoints. The union of the linear spans $\langle E\rangle$ for $E \in|A|$ is a $q$-dimensional variety of minimal degree.

(i) $\Longrightarrow$ (iii). If there exists $\bar{D} \in C_{e+1}^{1}$ satisfying $\left(\Delta_{g-e}\right)$ for some $1 \leq e \leq q$, then the locus

$$
W:=\left\{\bar{D}+x_{1}+\cdots+x_{g-(q+e)}: \bar{D} \in C_{e+1}^{1} \text { satisfying }\left(\Delta_{g-e}\right), x_{i} \in C\right\} \subset C_{g-q+1}
$$

consists of divisors not satisfying $\left(\Delta_{q}\right)$ and has dimension

$$
\operatorname{dim}(W) \geq g-(q+e)+1 \geq g-2 q+1 .
$$

Therefore $\bar{W}$ is a component of $C_{g-q+1}^{1}$, contradicting (i).

Remark 3.2. The proof of the implication (i) $\Longrightarrow$ (iii) fails if $g=2 q$. In fact, a general curve $C$ of genus $g=2 q$ has a primitive $g_{q+1}^{1}$ and $\left(\Delta_{q}\right)$ holds on $C$ in the strong sense. In this case $V_{q+1}^{q}\left(K_{C}\right)=C_{q+1}^{1}$ is reducible in several components of dimension 1: their number is given by Castelnuovo's formula [Arbarello et al. 1985, p. 211].

Remark 3.3. The implication (ii) $\Longrightarrow$ (i) does not hold. In fact, if $C$ is a bielliptic curve then $\operatorname{gon}(C)=4$. On the other hand, $C_{g-1}^{1}$ has two components [Teixidor i Bigas 1984], both having dimension $g-3$, equal to the expected dimension, but $\left(\Delta_{2}\right)$ holds only on one of them. Therefore, in this case the implication holds only in a weak sense.

Remark 3.4. If $\left(\Delta_{2}\right)$ holds then $\operatorname{Cliff}(C) \geq 2$. This has been proved in [Green and Lazarsfeld 1985] using Mumford-Martens. Note that they only assumed that $\left(\Delta_{2}\right)$ holds on some component of $C_{g-1}^{1}$. The implication $\left(\Delta_{3}\right)$ holds $\Longrightarrow \operatorname{gon}(C) \geq 5$ has been considered in [Voisin 1988]. In both cases $q=2,3$, the converse implication

$$
\operatorname{Cliff}(C) \geq q \Rightarrow\left(\Delta_{q}\right) \text { holds on some component of } C_{g-q+1}^{1}
$$

has also been proved. 
Remark 3.5. Assume $g$ is odd. On a general curve $C$ of Clifford dimension 1 there is a $D \in C_{(g+3) / 2}$ satisfying $\left(\Delta_{(g-1) / 2}\right)$. The reason is that $C$ has gonality $(g+3) / 2$, and a pencil computing its gonality is necessarily primitive. Therefore a divisor $D$ in the pencil satisfies $\left(\Delta_{(g-1) / 2}\right)$.

In the case $L=K_{C}$, Proposition 2.6 implies:

Proposition 3.6. Let $C$ be a curve of genus $g \geq 2 q+2$ such that the dimension of the locus $W_{g-q}^{1}(C)$ equals the expected dimension $g-2 q-2$ and $\operatorname{dim}\left(W_{g-q+2}^{2}(C)\right) \leq$ $g-2 q-2$. Then $\left(\Delta_{q}\right)$ holds on $C$ in the strong sense.

Proof. Since $\operatorname{dim}\left(W_{g-q}^{1}(C)\right)=g-2 q-2$, we obtain $\operatorname{dim}\left(V_{g-q}^{g-q-1}\left(K_{C}\right)\right)=g-2 q-1$, which is (2) of Proposition 2.6 in this case. From "excess linear series" it follows that the dimension of $W_{g-q+1}^{1}(C)$ also equals the expected dimension $g-2 q$, and hence $\operatorname{dim}\left(V_{g-q+1}^{g-q}\left(K_{C}\right)\right)=g-2 q+1$. Finally, $\operatorname{dim}\left(W_{g-q+2}^{2}(C)\right) \leq g-2 q-2$ implies that $\operatorname{dim}\left(V_{g-q+2}^{g-q}\left(K_{C}\right)\right) \leq g-2 q$, as $V_{g-q+2}^{g-q}\left(K_{C}\right)=C_{g-q+2}^{2}$. Hence all the conditions required in Proposition 2.6 are satisfied.

Remark 3.7. If the curve $C$ is of gonality $(q+1)$ or less, then the hypotheses of Proposition 3.6 are not satisfied. Indeed, if $A$ is a $g_{q+1}^{1}$, then $W_{g-q}^{1}(C)$ contains the variety $\{A\}+W_{g-2 q-1}(C)$, which is of dimension $g-2 q-1$.

If the curve $C$ is instead of gonality $(q+2)$, then the hypothesis that

$$
\operatorname{dim}\left(W_{g-q}^{1}(C)\right)=\rho(g, 1, g-q)=g-2 q-2
$$

coincides with the linear growth condition on the dimension of Brill-Noether loci, from [Aprodu 2005]. It was proved there that this condition implies Green's conjecture, i.e., condition $\left(M_{q}\right)$.

Remark 3.8. If $q=2$, and $C$ is neither trigonal, bielliptic nor plane quintic, the hypotheses of Proposition 3.6 are satisfied. Indeed, if one of the two fails, then we obtain a contradiction with the Mumford-Martens dimension theorem. Likewise, for $q=3$ the failure of the hypotheses contradicts Keem's dimension theorem [Voisin 1988, Proposition II.0].

Corollary 3.9. Assume that $g \geq 2 q+2, q \geq 2$, and that $\operatorname{dim}\left(C_{g-q+2}^{2}\right) \leq g-2 q$. If $\left(\Delta_{q+1}\right)$ holds on $C$ in the strong sense then $\left(\Delta_{q}\right)$ also holds on $C$ in the strong sense.

Applying Proposition 3.6 and Lemma 2.8, we obtain the following existence result:

Corollary 3.10. For a general triple $(C, L, D)$, with $L$ special and $D \in V_{r-q+2}^{r-q+1}(L)$, the condition $\left(\Delta_{q}\right)$ is satisfied.

The meaning of generality in the statement is that $L$ is a general projection of the canonical bundle, and hence the speciality index equals 1 . More precise existence results are proved by Coppens and Martens, and by Farkas; see [Farkas 2008, Theorem 0.5] and the references therein. 


\section{Condition $\left(\Delta_{q}\right)$ and geometry}

Proposition 4.1. Assume that $r \geq \max \{4,2 q-1\}$. Suppose that $L$ is special and condition $\left(\Delta_{q}\right)$ holds on $C$ with respect to $L$ in the strong sense. Then $\varphi_{L}(C) \subset \mathbb{P}^{r}$ is not contained in a $q$-dimensional variety of minimal degree $(r-q+1)$ unless $r=2 q-1$ and $C$ has a basepoint-free $g_{q+1}^{1}$.

Proof. Assume that $r \geq 2 q$. We note that $C$ has no $g_{q+1}^{1}$. Indeed, if we have a $g_{q+1}^{1}$, then $A+C_{r-2 q+1}$, with $A \in\left|g_{q+1}^{1}\right|$, fill up a component of $V_{r-q+2}^{r-q+1}(L)$, and any element of this locus fails condition (c) of the definition of $\left(\Delta_{q}\right)$.

Assume by contradiction that $\varphi_{L}(C) \subset X$, a $q$-dimensional variety of minimal degree $r-q+1$. Then $X$ is ruled by a one-dimensional family of $(q-1)$-planes. Let $\Lambda$ be a general such $(q-1)$-plane, and let $E=\Lambda \cap \varphi_{L}(C)$ and $n=\operatorname{deg}\left(\Lambda \cap \varphi_{L}(C)\right)$. Then $n \geq q+2$ by what we have just shown. Decompose $E=A+B$ with $\operatorname{deg}(A)=q+1$. Let $D=A+y_{1}+\cdots+y_{r-2 q+1}$ with the $y_{i}$ general points of $C$. Then $D \in V_{r-q+2}^{r-q+1}(L)$, but it does not satisfy $\left(\Delta_{q}\right)$. On the other hand, the divisor $D$ depends on $1+(r-2 q+1)=r-2 q+2$ parameters. Therefore it is a general point of a component of $V_{r-q+2}^{r-q+1}(L)$, a contradiction.

In the case $r=2 q-1$, the only possibility for $C$ to be on a variety of minimal degree is that $C$ have a basepoint-free $g_{q+1}^{1}$, and, in this case, $S_{q+1}(L)$ will have a rational component. The case when $X$ is a cone over the Veronese surface can be treated similarly, by general projection to $\mathbb{P}^{2 q-1}$ using Lemma 2.8 .

Note that if $C$ is contained in an $e$-dimensional variety of minimal degree $(r-e+1)$ with $e \leq q$, then it is contained also in a $q$-dimensional variety of minimal degree $(r-q+1)$ [Harris 1981].

As we will see, the validity of property $\left(M_{3}\right)$ is tightly connected with properties of surfaces of low degree in $\mathbb{P}^{5}$. As an illustration of the geometric content of Definition 2.3, we study surfaces of degree $n \leq 6$.

Proposition 4.2. Assume that $r=5, L$ is special and $\left(\Delta_{3}\right)$ holds on $C$ with respect to $L$ in the strong sense. Then $\varphi_{L}(C) \subset \mathbb{P}^{5}$ is not contained in a nonsingular surface of degree $\leq 6$ unless it has a $g_{4}^{1}$.

Proof. Assume that $C \subset S$, a nonsingular surface of degree $n \leq 6$. Consider the case $n=6$. The possibilities for a nonsingular surface of degree 6 in $\mathbb{P}^{5}$ are described in [Ionescu 1984], and are the following: (i) an elliptic scroll with sectional genus $g=1$ and $e=0$; (ii) a Castelnuovo surface with sectional genus $g=2$ defined by the embedding in $\mathbb{P}^{5}$ of the blow-up $X=\mathrm{B}_{p_{1}, \ldots, p_{6}, q}\left(\mathbb{P}^{2}\right)$ of $\mathbb{P}^{2}$ at seven general points via the very ample linear system $|\mathscr{L}|=\left|4 H-E_{1}-E_{2}-\cdots-E_{6}-2 A\right|$ (with the obvious notation) corresponding to the system of plane quartics passing simply through $p_{1}, \ldots, p_{6}$ and doubly through $q$. 
In case (i), let $\ell \subset S$ be a general line of the ruling, and let $k=\operatorname{deg}\left(\mathscr{O}_{C}(\ell)\right)$. Then $k \geq 2$, and if $k=2$ then $C$ is bielliptic, so it has a $g_{4}^{1}$. If $k \geq 3$, then adding a general $p \in C$ to a subdivisor of degree 3 of $\mathscr{O}_{C}(\ell)$ we obtain an element of $S_{4}(L)$ which does not satisfy $\left(\Delta_{3}\right)$ and which depends on two parameters, a contradiction.

In case (ii), the system $|H-A|$ is a pencil of conics on the surface $S$. The divisors $D \in\left|\mathcal{O}_{C}(H-A)\right|$ have degree say $m \geq 3$ and $\operatorname{dim}|D| \geq 1$. If $m \leq 4$ then $C$ has a $g_{4}^{1}$. Otherwise the divisors $D$ contain subdivisors of degree 4 contradicting the other conditions.

If $n=5$, then $S$ is a Del Pezzo surface. Let $|\gamma|$ be a pencil of conics on $S$ and let $N=\mathscr{O}_{C}(\gamma)$. Then $N$ gives a $g_{4}^{1}$ or contradicts $\left(\Delta_{3}\right)$, depending on whether $\operatorname{deg}(N) \leq 4$ or $\operatorname{deg}(N) \geq 5$.

If $n=4$, the conclusion follows from Proposition 4.1.

\section{Condition $\left(\Delta_{3}\right)$ and Koszul cohomology}

In this section, we briefly recall the relation between Koszul cohomology and vector bundles, as well as the definition of syzygy schemes.

Consider $X$ a smooth projective variety, and let $L$ be a globally generated line bundle on $X$. We let

$$
\varphi_{L}: X \rightarrow \mathbb{P}\left(H^{0}(L)^{\vee}\right) \cong \mathbb{P}^{r}, \quad r+1=h^{0}(L)
$$

be the morphism defined by $L$.

We have an exact sequence

$$
0 \longrightarrow M_{L} \longrightarrow H^{0}(L) \otimes \mathrm{O}_{X} \longrightarrow L \longrightarrow 0,
$$

where $M_{L}=\varphi^{*}\left(\Omega_{\mathbb{p} r}(1)\right)$ is locally free of rank $r$. If $r=1$, i.e., if $|L|$ is a basepointfree pencil, then $M_{L}=L^{-1}$. Taking the $n$-th exterior power $(1 \leq n \leq r)$ we obtain the exact sequence

$$
0 \longrightarrow \bigwedge^{n} M_{L} \longrightarrow \bigwedge^{n} H^{0}(L) \otimes O_{X} \longrightarrow \bigwedge^{n-1} M_{L} \otimes L \longrightarrow 0 .
$$

For any coherent sheaf $\mathscr{F}$ on $X$, twisting the sequence above with $\mathscr{F}$, with powers of $L$ and taking global sections, we obtain isomorphisms

$$
K_{n, m}(X, \mathscr{F} ; L) \cong \operatorname{Coker}\left\{\bigwedge^{n+1} H^{0}(L) \otimes H^{0}\left(\mathscr{F} \otimes L^{m-1}\right) \rightarrow H^{0}\left(\bigwedge^{n} M_{L} \otimes \mathscr{F} \otimes L^{m}\right)\right\} .
$$

The syzygy schemes were introduced and studied in [Green 1984; Ehbauer 1994]. The idea behind the definition of syzygy schemes is that one reason for which a linearly normal curve $C$ in $\mathbb{P}^{r}$ has some nonvanishing $K_{n, 1}$ is that $C$ lies on a variety of special type. The varieties under question are cut out by quadrics; more precisely, by the quadrics involved in syzygies. 
The general set-up is the following. Let $C$ be a smooth curve, $L$ a globally generated (preferably very ample) line bundle on $C$ and set $V=H^{0}(L)$. Start with the short exact sequence of sheaves on the projective space

$$
0 \longrightarrow \mathscr{I}_{C} \longrightarrow \mathrm{O}_{\mathrm{pr}} \longrightarrow \mathrm{O}_{C} \longrightarrow 0 \text {. }
$$

Note that for any $n$ and $m$ we have $K_{n, m}\left(\mathbb{P}^{r}, \mathscr{O}_{C} ; \mathcal{O}_{\mathbb{P r}}(1)\right) \cong K_{n, m}(C ; L)$.

Taking Koszul cohomology with respect to Opr (1), and using the vanishing of Koszul cohomology on the projective space, we obtain isomorphisms

$$
K_{n, m}(C ; L) \cong K_{n-1, m+1}\left(\mathbb{P}^{r}, \Phi_{C} ; \mathscr{O}_{\mathbb{P r}}(1)\right),
$$

for any $n$ and $m$ except for the cases $(n, m)=(0,0)$ or $(n, m)=(1,-1)$. On the other hand, from the general description of mixed Koszul cohomology, we know that

$$
\begin{aligned}
K_{n-1, m+1}\left(\mathbb{P}^{r}, \Phi_{C} ; O_{\mathbb{p r}}\right. & (1)) \\
& \cong \operatorname{Coker}\left\{\bigwedge^{n} V \otimes H^{0}\left(\Phi_{C}(m)\right) \rightarrow H^{0}\left(\Omega_{\mathbb{P r}}^{n-1}(n+m) \otimes \Phi_{C}\right)\right\} .
\end{aligned}
$$

Observe that for the case $m=1$ we have $H^{0}\left(\Phi_{C}(m)\right)=0$, and hence we obtain an isomorphism

$$
K_{n, 1}(C ; L) \cong H^{0}\left(\Omega_{\mathbb{P r}}^{n-1}(n+1) \otimes \mathscr{I}_{C}\right) ;
$$

in particular, any nonzero Koszul cohomology class $\alpha \in K_{n, 1}(C ; L)$ corresponds to a section in $H^{0}\left(\Omega_{\mathbb{p r}}^{n-1}(n+1)\right)$ vanishing along $C$. The zero-scheme of this section is called the syzygy scheme associated to $\alpha$, and is denoted by $\operatorname{Syz}(\alpha)$. Note that a syzygy scheme is cut out by quadrics, as the sheaf $\Omega_{\mathbb{p r}}^{n-1}(n+1)$ is a subsheaf of $\bigwedge^{n-1} V \otimes \mathcal{O}_{\mathbb{p} r}(2)$. The scheme-theoretic intersection of all the syzygy schemes is denoted by $\operatorname{Syz}_{n}(C)$. It contains $C$ and is cut out by quadrics as well.

We record next two remarkable classification results concerning syzygy schemes, due to Green and Ehbauer.

Theorem 5.1 (Green's $K_{p, 1}$ ). If $K_{r-1,1}(C, L) \neq 0$, then $C$ is a rational normal curve and $\operatorname{Syz}_{r-1}(C)=C$. If $C$ is of degree $\geq r+2$ and $K_{r-2,1}(C, L) \neq 0$, then $\mathrm{Syz}_{r-2}(C)$ is a surface of minimal degree $(r-1)$.

Theorem 5.2 (Ehbauer). If $C$ has degree $\geq r+13$ and $K_{r-3,1}(C, L) \neq 0$, then $\operatorname{Syz}_{r-3}(C)$ is either a surface of minimal degree $(r-1)$, a surface of degree $r$ or a threefold of minimal degree $(r-2)$.

We recall the following:

Definition 5.3. The line bundle $L$ has property $\left(M_{q}\right)$ if $K_{n, 1}(C ; L)=0$ for all $n \geq r-q$.

We prove: 
Theorem 5.4. Assume $g \geq 14, r \geq 5$, $L$ is very ample and special of degree $\geq r+13$, and $\left(\Delta_{3}\right)$ holds on $C$ with respect to $L$ in the strong sense. Then L satisfies $\left(M_{3}\right)$ unless $\operatorname{gon}(C) \leq 4$.

Proof. Applying Ehbauer's characterisation of syzygy schemes, if $L$ fails property $\left(M_{3}\right)$, then $C$ lies either on a surface of minimal degree, on a threefold of minimal degree or on a surface of degree $r$. The first two cases are excluded by Proposition 4.1. Projecting generically to $\mathbb{P}^{5}$ and applying Lemma 2.8 and Proposition 4.2, we see that $C$ cannot lie on a smooth surface of degree 5 . If it lies on a singular surface of degree 5 in $\mathbb{P}^{5}$, then, projecting from a singular point, the curve in $\mathbb{P}^{4}$ lies on a surface of minimal degree. In particular, since the curve is of gonality $\geq 5$, the image of $C$ in $\mathbb{P}^{4}$ has a $k$-secant line for some $k \geq 5$, and hence the image of $C$ in $\mathbb{P}^{5}$ has a one-dimensional family of $k$-secant 2-planes with $k \geq 5$, which contradicts the assumptions.

Remark 5.5. The same argument together with Green's $K_{p, 1}$-theorem gives a similar statement for the weaker property $\left(M_{2}\right)$.

\section{References}

[Aprodu 2005] M. Aprodu, "Remarks on syzygies of $d$-gonal curves", Math. Res. Lett. 12:2-3 (2005), 387-400. MR 2006d:14028 Zbl 1084.14032

[Aprodu and Nagel 2010] M. Aprodu and J. Nagel, Koszul cohomology and algebraic geometry, University Lecture Series 52, American Mathematical Society, Providence, RI, 2010. MR 2011f:14051 Zbl 1189.14001

[Arbarello and Sernesi 1978] E. Arbarello and E. Sernesi, "Petri's approach to the study of the ideal associated to a special divisor", Invent. Math. 49:2 (1978), 99-119. MR 80c:14020 Zbl 0399.14019

[Arbarello et al. 1985] E. Arbarello, M. Cornalba, P. A. Griffiths, and J. Harris, Geometry of algebraic curves, I, Grundlehren der Mathematischen Wissenschaften 267, Springer, New York, 1985. MR 86h:14019 Zbl 0559.14017

[Teixidor i Bigas 1984] M. Teixidor i Bigas, “For which Jacobi varieties is Sing $\Theta$ reducible?”, J. Reine Angew. Math. 354 (1984), 141-149. MR 86c:14025 Zbl 0542.14020

[Ehbauer 1994] S. Ehbauer, "Syzygies of points in projective space and applications", pp. 145-170 in Zero-dimensional schemes (Ravello, 1992), edited by F. Orecchia and L. Chiantini, de Gruyter, Berlin, 1994. MR 95h:13012 Zbl 0840.14032

[Farkas 2008] G. Farkas, "Higher ramification and varieties of secant divisors on the generic curve", J. Lond. Math. Soc. (2) 78:2 (2008), 418-440. MR 2010f:14029 Zbl 1155.14024

[Farkas 2009] G. Farkas, "Koszul divisors on moduli spaces of curves", Amer. J. Math. 131:3 (2009), 819-867. MR 2010f:14030 Zbl 1176.14006

[Green 1984] M. L. Green, "Koszul cohomology and the geometry of projective varieties", J. Differential Geom. 19:1 (1984), 125-171. MR 85e:14022 Zbl 0559.14008

[Green and Lazarsfeld 1985] M. Green and R. Lazarsfeld, "A simple proof of Petri's theorem on canonical curves", pp. 129-142 in Geometry today (Rome, 1984), edited by E. Arbarello et al., Progr. Math. 60, Birkhäuser, Boston, 1985. MR 88h:14038 Zbl 0577.14018 
[Green and Lazarsfeld 1986] M. Green and R. Lazarsfeld, "On the projective normality of complete linear series on an algebraic curve", Invent. Math. 83:1 (1986), 73-90. MR 87g:14022 Zbl 0594.14010

[Harris 1981] J. Harris, "A bound on the geometric genus of projective varieties", Ann. Scuola Norm. Sup. Pisa Cl. Sci. (4) 8:1 (1981), 35-68. MR 82h:14010 Zbl 0467.14005

[Ionescu 1984] P. Ionescu, "Embedded projective varieties of small invariants", pp. 142-186 in Algebraic geometry (Bucharest, 1982), edited by L. Bădescu and D. Popescu, Lecture Notes in Math. 1056, Springer, Berlin, 1984. MR 85m:14024 Zbl 0542.14024

[Petri 1925] K. Petri, “Über Spezialkurven, I”, Math. Ann. 93:1 (1925), 182-209. MR 1512232 Zbl 51.0510.01

[Saint-Donat 1973] B. Saint-Donat, "On Petri's analysis of the linear system of quadrics through a canonical curve”, Math. Ann. 206 (1973), 157-175. MR 49 \#2752 Zbl 0315.14010

[Schreyer 1991] F.-O. Schreyer, "A standard basis approach to syzygies of canonical curves", J. Reine Angew. Math. 421 (1991), 83-123. MR 92j:14040 Zbl 0729.14021

[Voisin 1988] C. Voisin, "Courbes tétragonales et cohomologie de Koszul”, J. Reine Angew. Math. 387 (1988), 111-121. MR 89e:14036 Zbl 0652.14012

Communicated by David Eisenbud

Received 2014-04-25 Revised 2015-01-27 Accepted 2015-03-02

marian.aprodu@imar.ro

Simion Stoilow Institute of Mathematics of the Romanian Academy, P.O. Box 1-764, 014700 Bucharest, Romania

Faculty of Mathematics and Computer Science, University of Bucharest, 14 Academiei Street, 010014 Bucharest, Romania

sernesi@mat.uniroma3.it

Dipartimento di Matematica e Fisica, Università degli Studi Roma Tre, Largo San Leonardo Murialdo, I-00146 Roma, Italy 


\section{Algebra \& Number Theory}

msp.org/ant

\section{EDITORS}

MANAGING EDITOR

Bjorn Poonen

Massachusetts Institute of Technology

Cambridge, USA

\author{
EDITORIAL BOARD CHAIR \\ David Eisenbud \\ University of California \\ Berkeley, USA
}

\section{BOARD OF EDITORS}

Georgia Benkart

Dave Benson

Richard E. Borcherds

John H. Coates

J-L. Colliot-Thélène

Brian D. Conrad

Hélène Esnault

Hubert Flenner

Edward Frenkel

Andrew Granville

Joseph Gubeladze

Roger Heath-Brown

Craig Huneke

János Kollár

Yuri Manin

Barry Mazur

Philippe Michel
University of Wisconsin, Madison, USA

University of Aberdeen, Scotland

University of California, Berkeley, USA

University of Cambridge, UK

CNRS, Université Paris-Sud, France

University of Michigan, USA

Freie Universität Berlin, Germany

Ruhr-Universität, Germany

University of California, Berkeley, USA

Université de Montréal, Canada

San Francisco State University, USA

Oxford University, UK

University of Virginia, USA

Princeton University, USA

Northwestern University, USA

Harvard University, USA

École Polytechnique Fédérale de Lausanne
Susan Montgomery

Shigefumi Mori

Raman Parimala

Jonathan Pila

Anand Pillay

Victor Reiner

Peter Sarnak

Joseph H. Silverman

Michael Singer

Vasudevan Srinivas

J. Toby Stafford

Ravi Vakil

Michel van den Bergh

Marie-France Vignéras

Kei-Ichi Watanabe

Efim Zelmanov

Shou-Wu Zhang
University of Southern California, USA

RIMS, Kyoto University, Japan

Emory University, USA

University of Oxford, UK

University of Notre Dame, USA

University of Minnesota, USA

Princeton University, USA

Brown University, USA

North Carolina State University, USA

Tata Inst. of Fund. Research, India

University of Michigan, USA

Stanford University, USA

Hasselt University, Belgium

Université Paris VII, France

Nihon University, Japan

University of California, San Diego, USA

Princeton University, USA

\section{PRODUCTION}

production@msp.org

Silvio Levy, Scientific Editor

See inside back cover or msp.org/ant for submission instructions.

The subscription price for 2015 is US $\$ 255 /$ year for the electronic version, and $\$ 440 /$ year ( $+\$ 55$, if shipping outside the US) for print and electronic. Subscriptions, requests for back issues and changes of subscribers address should be sent to MSP.

Algebra \& Number Theory (ISSN 1944-7833 electronic, 1937-0652 printed) at Mathematical Sciences Publishers, 798 Evans Hall \#3840, c/o University of California, Berkeley, CA 94720-3840 is published continuously online. Periodical rate postage paid at Berkeley, CA 94704, and additional mailing offices.

ANT peer review and production are managed by EditFLOW ${ }^{\circledR}$ from MSP.

\section{PUBLISHED BY}

- mathematical sciences publishers

nonprofit scientific publishing

http://msp.org/

(C) 2015 Mathematical Sciences Publishers 


\section{Algebra \& Number Theory}

Volume $9 \quad$ No. $3 \quad 2015$

Hurwitz monodromy and full number fields

DAVID P. ROBERTS and AKSHAY VENKATESH

The characteristic polynomial of the Adams operators on graded connected Hopf algebras

Marcelo Aguiar and Aaron Lauve

Secant spaces and syzygies of special line bundles on curves

MARIAN APRODU and EDOARDO SERNESI

Complex group algebras of the double covers of the symmetric and alternating groups

601

Christine Bessenrodt, Hung Ngoc Nguyen, Jørn B. Olsson and Hung P. TONG-VIET

Fano schemes of determinants and permanents

Melody Chan and NATHan Ilten

Triple intersection formulas for isotropic Grassmannians

VIJAY RAVIKUMAR

On the basepoint-free theorem for log canonical threefolds over the algebraic closure of a finite field

Diletta Martinelli, Yusuke NaKamura and Jakub Witaszek

The torsion group of endotrivial modules

JON F. CARLSON and JACQUES THÉVENAZ 